\title{
Thermal hydraulic efficiency of a single diaphragm established with a clearance in a circular tube
}

\author{
Tatyana Bogatko ${ }^{1, *}$, and Viktor Terekhov ${ }^{1}$ \\ ${ }^{1}$ Kutateladze Institute of Thermophysics, 630090 Novosibirsk, Russia.
}

\begin{abstract}
Results of a numerical study of the flow structure and turbulent heat transfer in a round tube with a mounted single diaphragm with height $\mathrm{h}$ and some clearance $\mathrm{c}$ between the tube and the diaphragm. The size of the clearance between the diaphragm and the tube wall was varied within range $\mathrm{A}=\mathrm{c} / \mathrm{h}=0-0.33$. It was determined that an increase in the gap between the diaphragm and the tube wall alters the structure of the recirculation region and eliminate the stagnation zones in the region of secondary vortex. The flow regime, when the values of average heat transfer behind the diaphragm in the presence of a clearance exceed those for the attached rib, was distinguished. When increasing the gap height from $\mathrm{A}=0$ to 0.33 , the thermal enhancement factor increases by $30 \%$.
\end{abstract}

\section{Introduction}

Study of the flow structure and heat and mass transfer behind a flat obstacle and the system of such obstacles were relatively extensive since the 60 s of the last century. Attention the study of the features of the flow around detached ribs was attracted much later. The idea of their use for heat transfer improvement is based on formation of the wall jet flow, which destroys the stagnation zone directly behind an obstacle. We can note only a few papers of the experimental and calculation nature devoted to this field [1-6]. All of them were carried out mainly for the rectangular channels and disconnected square obstacles. The exception is a series of works [7-9], where development of the flow with disconnected diaphragms in a rounds tube was studied, and periodic destruction of the flow was performed by the rings of a round wire or twisted ribbon. Alternation of connected and disconnected obstacles along the channel leads to additional possibility of changing the integral heat transfer coefficient [10].

The detailed experimental studies were carried out in [2-3], where they studied the effect of Reynolds number, obstacle sizes and relative height of the clearance on the flow structure and turbulent heat transfer in a rectangular channel with the ribbed wall. Investigation results show that the thermohydraulic efficiency at this way of heat transfer enhancement is significantly higher than the use of attached ribs.

${ }^{*}$ Corresponding author: tbogatko@gmail.com 
As can be seen from the above, the mechanism of heat transfer enhancement in the channels with disconnected obstacles is studied insufficiently. This is explained by the multifactor character of the complex heat transfer process, three-dimensional nature of the flow, and the features of the vortex flow formation in the rectangular channels with different numbers of ribbed walls in comparison with the round tubes. Moreover, additional difficulties in analyzing are caused by interference of separated flows from the alternating obstacles. In this connection it is necessary to study in detail the flow structure under the simpler conditions of the flow around a single two-dimensional obstacles with varying disconnection parameter $\mathrm{A}=\mathrm{c} / \mathrm{h}$, where $\mathrm{c}$ is the size of a clearance between the diaphragm and the tube wall, and $\mathrm{h}$ is the height of the diaphragm (Fig. 1). At that, it is important to determine the effect of parameter $\mathrm{A}$ on the local and integral characteristics of heat transfer and resistance. These data can serve the basis for the analysis of more complex transfer processes in channels with the system of obstacles.

\section{Flow scheme. Calculation method}

The scheme of computational domain is shown in Fig. 1. A flat annular diaphragm is placed into the cylindrical channel with the diameter of $100 \mathrm{~mm}$ and length of $900 \mathrm{~mm}$ at the distance of $100 \mathrm{~mm}$ from the inlet. Its height was constant $\mathrm{h}=15 \mathrm{~mm}$, and the size of the clearance was varied in range $\mathrm{c}=0-5 \mathrm{~mm}$. The velocity profile at the tube inlet was uniform, the working medium was air of $10^{\circ} \mathrm{C}$. A change in thermal-physical properties was neglected in calculations. The Reynolds number, calculated by the tube diameter and the mean mass velocity, was constant $\mathrm{Re}=27500$.

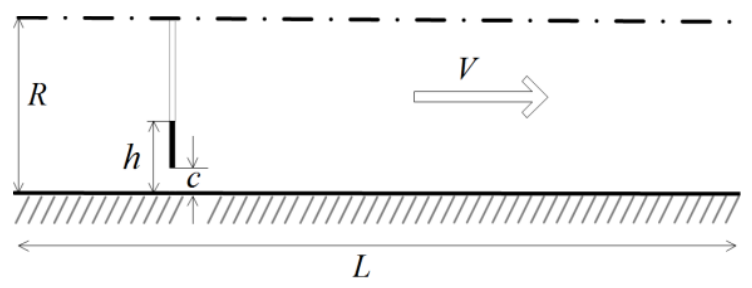

Fig. 1. Scheme of the computational domain.

The calculations were carried out in the framework of the model of incompressible liquid based on the system of stationary Navier-Stokes and energy equations, Reynolds averaged (RANS). The main tool for the study is the universal calculation complex FLUENT. The problem statement is two-dimensional; the flow is stationary and axisymmetrical. The turbulence model k- $\omega$ SST is chosen for calculations [11], as the most appropriate for calculation of turbulent separated flows. The previous studies of the authors prove also that for the models, implemented in the given set, the results obtained with application of the mentioned model are the best to match the physics of the given flow type.

\section{Calculation results}

The flow structure near the disconnected rib is shown in Fig.2. The field of longitudinal velocity component is shown by the colour; the streamlines are shown by the vectors. 
a)

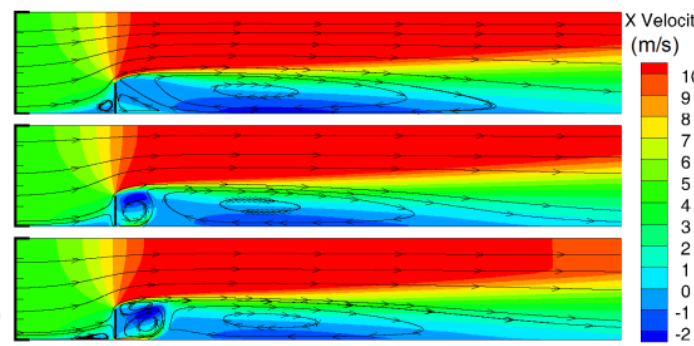

d)

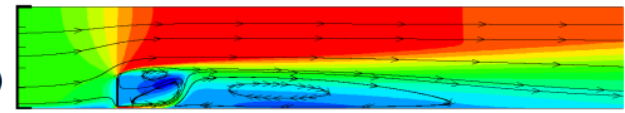

e)

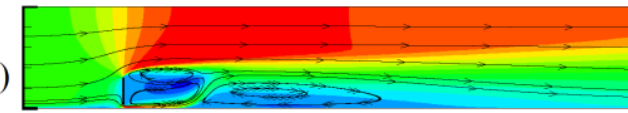

f)

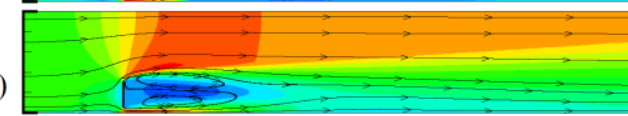

g)

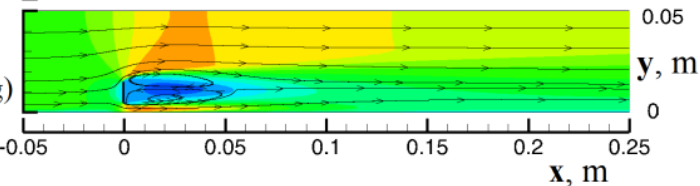

Fig. 2. The stream lines and the longitudinal component of the velocity field in the area of the diaphragm. a) $\mathrm{c} / \mathrm{h}=0$, attached diaphragm; b) c/h $=0.03$; c) 0.07 ; d) 0.1 ; e) 0.13 ; f) $0.2 ;$ g) 0.33 .

brings significant changes in the structure of the recirculation region. The area of reverse brings significant changes in the structure of the recirculation region. The area of reverse flows, representing a pair of counter-rotating vortices, is formed behind a rib like behind a poorly streamlined body. At that, the velocity in the flow core decreases significantly.

It is interesting to consider the transitional regime, when a pair of vortices just starts to be formed, and a separation bubble reduces in size and moves downstream. One can observe this flow regime in Fig. 2-c-e, at the size of a clearance between the tube wall and diaphragm of 1-2 mm. Already at $\mathrm{c}=3 \mathrm{~mm}$ the above picture is observed, when the pair of equal vortices replaces the primary and secondary vortices in the recirculation zone.

Distribution of the surface friction coefficients on the tube wall (Fig.4) also show a considerable reduction of the recirculation zone. At that, the reverse flow area along the tube wall degenerates significantly for the large clearance values $\mathrm{c} / \mathrm{h}=0.2-0.33$. Actually, two vortices directed oppositely to the center of the recirculation zone remain behind the rib.

These features of the effect of parameter $\mathrm{c} / \mathrm{h}$ also affect the behaviour of the surface friction coefficient, whose calculation results are presented in Fig. 4. The largest size of the zone of reverse flows, where the friction is negative, is observed at flow separation behind the attached obstacle $(\mathrm{c} / \mathrm{h}=0)$. Respectively, the value of the friction coefficient for the attached obstacle recovers slower.

Distributions of the friction coefficient (Fig. 4) demonstrate that behind the separated rib with large gaps $\mathrm{c} / \mathrm{h}=0.2$ and 0.33 the separation zone has the different structure, and the reverse flows are not formed along the tube wall. That is why in Fig. 5, which shows the relative length of the recirculation region, the value in these two cases is zero $\left(X_{R} / h=0\right)$. The attachment point here was determined as the coordinate, where the surface friction on the wall is zero. Calculation results shown in Fig. 5 clearly demonstrate the influence of the 
clearance height on the size of recirculation zone. It can be seen that the length of a separation bubble is inversely proportional to the height of the clearance between the diaphragm and tube wall.

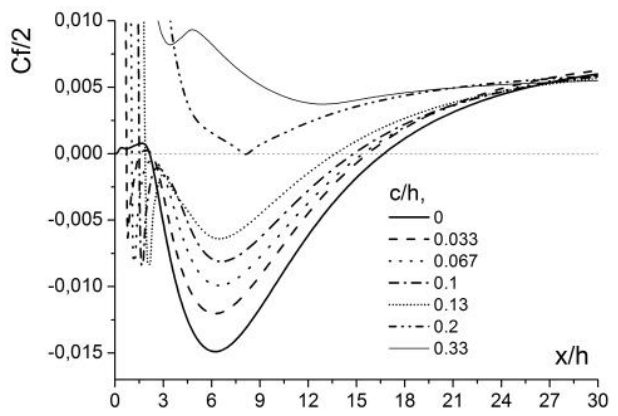

Fig. 4. The distribution of the friction coefficient on the tube wall behind the obstacle.

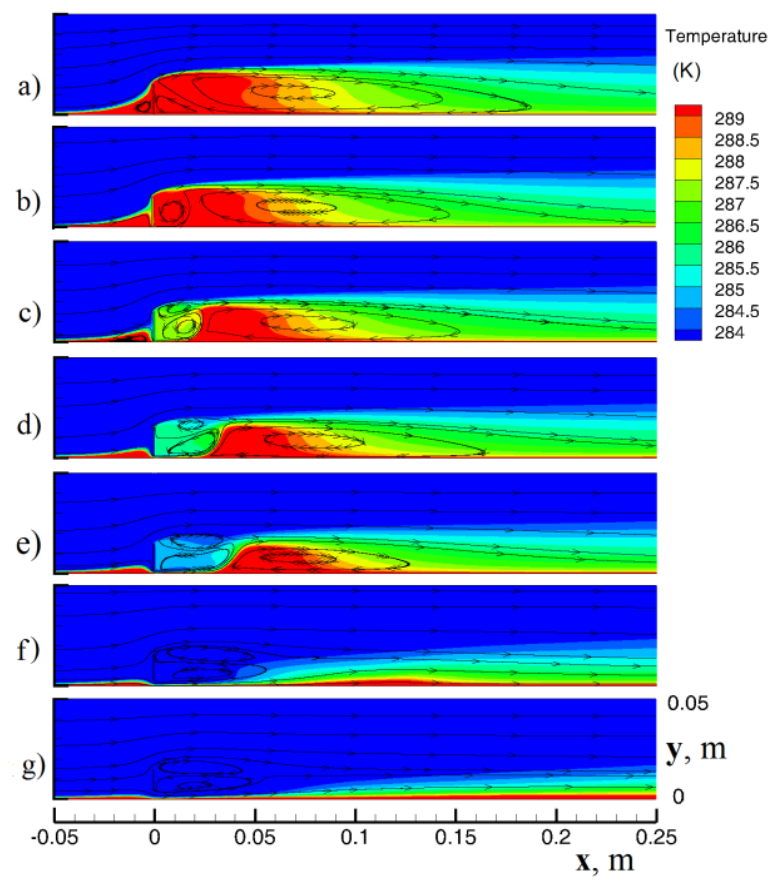

Fig.6. The stream lines and temperature field around the diaphragm. a) $\mathrm{A}=0$, attached diaphragm; b) 0.03 ; c) 0.07 ; d) 0.1 ; e) 0.13 ; f) 0.2 ; g) 0.33 .

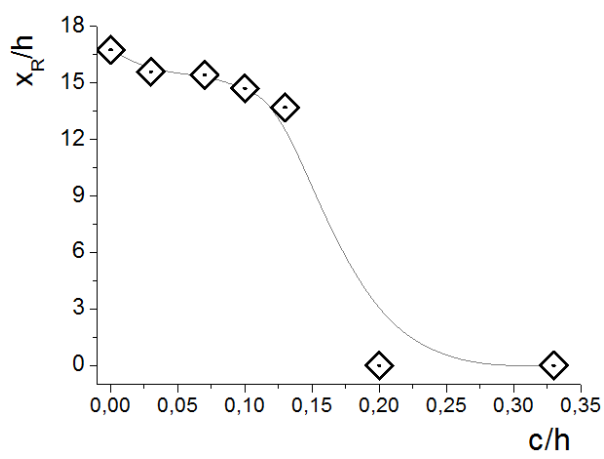

Fig. 5. Relative coordinate of the reattachment point.

The temperature fields at the flow around a rib, removed at the different distances from the wall, are shown in Fig.6. Here, we can clearly see the stagnation zone with the increased temperature values (Fig. 6 a). It is obvious that with an increase in the clearance between the diaphragm and tube wall, this zone is removed downstream (Fig. 6 b e) and degenerates gradually (Fig. $6 \mathrm{f}-\mathrm{g}$ ).

When choosing the optimal shape of an obstacle for the purposes of heat transfer intensification, the determining parameters are the integral heat transfer coefficient, total hydraulic losses and thermohydraulic efficiency. The last parameter represents the ratio of heat transfer gain to the power increase, spent to overcome the increased hydraulic losses. In this paper, the calculation of the average heat transfer coefficient was determined by integrating the local distributions in the area, which covers the distance of 5 heights to the obstacle, zone directly under the obstacle, and distance of 25 heights after the obstacle $(-5 \leq \mathrm{x} / \mathrm{h} \leq 25)$.

The results of calculation of the integral Nusselt number $\mathrm{Nu}_{\mathrm{sr}}$ for different values of the studied parameters are shown in Fig. 11. Data are presented in the relative form, where $\mathrm{Nu}_{0}$ is heat transfer in a smooth tube along the stabilized region. 
When considering the average values of heat transfer in the specified region (Fig. 7), the maximum is observed at $\mathrm{c} / \mathrm{h}=0.03$. This is caused by the fact that at the clearance height of $0.5 \mathrm{~mm}$, the recirculation zone remains almost of the same size as in the case of

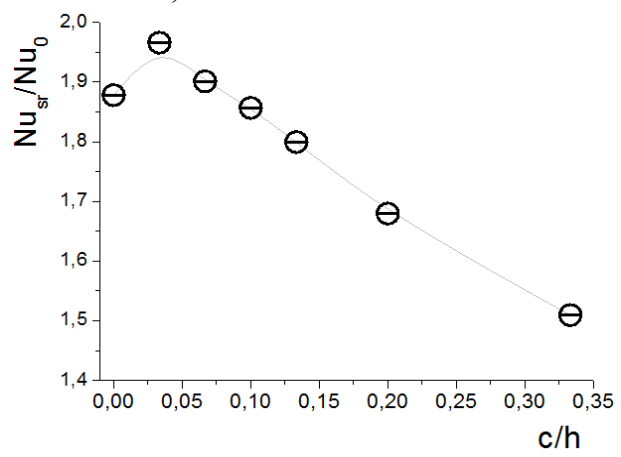

Fig.7. The relative values6 of the average heat transfer coefficient.

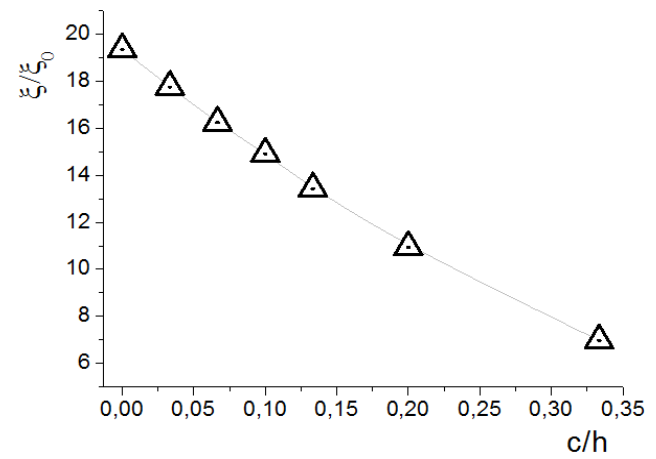

Fig. 8. The relative values of the coefficient of hydraulic losses.

theattached rib, but the formed wall jet gives the increased heat removal in the region under the diaphragm. Figure 8 shows the data for the hydraulic friction coefficient reduced to this value for a smooth tube.

We see that an increase in the scale of wall jet affects significantly investigation results. It is worth noting that with an increase in clearance height c from 0 to $5 \mathrm{~mm}$, the area of the

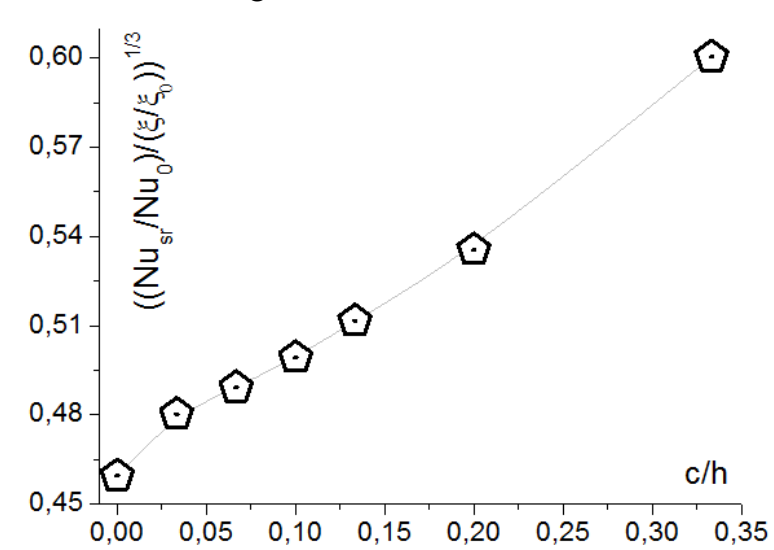

Fig. 9. Thermal enhancement factor depending on the height of the clearance between the diaphragm and the wall of the tube. obstacle surface obstruction changes by the factor of 1.6. A decrease in the drag associated with the above fact also contributes significantly to the overall result. Finally, we observe the three-fold decrease in resistance with variation of the tested parameter from 0 to 0.33 .

Data of thermal enhancement factor for different values of tested parameter $\mathrm{c} / \mathrm{h}$ are shown in Fig. 9. As it can be seen from these data, with an increase in the clearance between the diaphragm and tube wall from 0 to $5 \mathrm{~mm}$, thermal enhancement factor increases by $30 \%$. Basically, this occurs due to a significant reduction in the coefficient of hydraulic losses (Fig. 8) because the relative value of $\mathrm{Nu}_{\mathrm{sr}} / \mathrm{Nu}_{0}$ in this region of tube reduces only by $20 \%$. It should be specially emphasized that an obstacle studied here gives the high values of hydraulic resistance and cannot be used directly as a heat transfer intensifier. However, the comparative analysis and identified flow and heat transfer features for the obstacles with different values of parameter $\mathrm{c} / \mathrm{h}$ can be useful when selecting and optimizing the surfaces with intensified heat exchange. 


\section{Conclusion}

The turbulent structure and heat transfer behind a flat diaphragm in a round tube were studied numerically with variation of a clearance between the tube wall and diaphragm A $=\mathrm{c} / \mathrm{h}=0-0.33$.

It is shown that a wall jet formed in the slot leads to such a strong deformation of separated flow behind the diaphragm that for the large scale of the jet $(\mathrm{A}>0.2)$ there is no repeated attachment of the flow. Due to the breakdown of recirculation zone, the level of kinetic energy decreases and it is taken downstream by the jet.

It is found out that an increase in the distance between the diaphragm and tube wall reduces maximal heat transfer significantly.

The integral characteristics show that that for clearance values $\mathrm{c} / \mathrm{h}=0.033$ and 0.067 heat transfer becomes more intensive due to the increased heat removal on the tube wall under the diaphragm.

The pressure losses decrease by the factor of 3 at a change in the wall jet size from $\mathrm{c} / \mathrm{h}=$ 0 to 0.33 . At that, thermal enhancement factor increases by $30 \%$.

This work was supported by the Russian President's grant NSh-8780.2016.8.

\section{References}

1. J.P. Tsia, J.J. Hwang, Int. J. Heat Mass Transfer 42, 2071 (1999)

2. T.M. Liou, C.P. Yang, H.L. Lee, ASME J. Heat Transfer 119, 383 (1997)

3. T.M. Liou, S.H. Chen, International Int. J. Heat Mass Transfer 41, 1795 (1998)

4. A. Valencia, Numer. Heat Transfer, Part A. 37, 289 (2000)

5. J. Ahn, J.S. Lee, Int. J. Heat Mass Transfer 53, 445 (2010)

6. S. Eiamsa-ard, W. Changcharoen, Heat transfer - Asian Research 40, 20357 (2011)

7. C. Thianpong, K. Yongsiri, K. Nanan, S. Eiamsa-ard International Communications in Heat and Mass Transfer 39, 861 (2012)

8. V. Ozceyhan, S. Gunes, O. Buyukalaca, N. Altuntop, Applied Energy 83, 280 (2006)

9. K. Nanana, M. Pimsarnb, W. Jedsadaratanachaib, S. Eiamsa-arda, Int. Commun. Heat Mass 48, 133 (2013)

10. Jenn-Jiang Hwang, Chung-Hsin Chao, J. Thermophys. Heat Transf. 14, 579, (2000)

11. F.R. Menter, AIAA J. 32, 269 (1994) 